\title{
BOUNDARY DATA MAPS AND KREIN'S RESOLVENT FORMULA FOR STURM-LIOUVILLE OPERATORS ON A FINITE INTERVAL
}

\author{
Stephen Clark, Fritz Gesztesy, Roger Nichols and MaXim Zinchenko
}

\begin{abstract}
We continue the study of boundary data maps, that is, generalizations of spectral parameter dependent Dirichlet-to-Neumann maps for (three-coefficient) Sturm-Liouville operators on the finite interval $(a, b)$, to more general boundary conditions, began in [8] and [17]. While these earlier studies of boundary data maps focused on the case of general separated boundary conditions at $a$ and $b$, the present work develops a unified treatment for all possible self-adjoint boundary conditions (i.e., separated as well as non-separated ones).

In the course of this paper we describe the connections with Krein's resolvent formula for self-adjoint extensions of the underlying minimal Sturm-Liouville operator (parametrized in terms of boundary conditions), with some emphasis on the Krein extension, develop the basic trace formulas for resolvent differences of self-adjoint extensions, especially, in terms of the associated spectral shift functions, and describe the connections between various parametrizations of all self-adjoint extensions, including the precise relation to von Neumann's basic parametrization in terms of unitary maps between deficiency subspaces.
\end{abstract}

Mathematics subject classification (2010): Primary 34B05, 34B27, 34L40; Secondary 34B20, 34L05, 47A10, 47E05.

Keywords and phrases: Self-adjoint Sturm-Liouville operators on a finite interval, boundary data maps, Krein-type resolvent formulas, spectral shift functions, perturbation determinants, parametrizations of self-adjoint extensions.

\section{REFERENCES}

[1] N. I. Akhiezer And I. M. Glazman, Theory of Linear Operators in Hilbert Space, Volume II, Pitman, Boston, 1981.

[2] A. Alonso And B. Simon, The Birman-Krein-Vishik theory of selfadjoint extensions of semibounded operators, J. Operator Th. 4 (1980), 251-270; Addenda: 6 (1981), 407.

[3] N. ARonszajn And W. F. Donoghue, On exponential representations of analytic functions in the upper half-plane with positive imaginary part, J. Analyse Math. 5 (1956-57), 321-388.

[4] M. S. Ashbaugh, F. Gesztesy, M. Mitrea, R. Shterenberg, and G. Teschl, The Kreinvon Neumann extension and its connection to an abstract buckling problem, Math. Nachr. 283 (2010), $165-179$.

[5] M. S. Ashbaugh, F. Gesztesy, M. Mitrea, And G. Teschl, Spectral theory for perturbed Krein Laplacians in nonsmooth domains, Adv. Math. 223 (2010), 1372-1467.

[6] J. BEhrndt AND A. Luger, On the number of negative eigenvalues of the Laplacian on a metric graph, J. Phys. A: Math. Theor. 43 (2010), 474006.

[7] J. Behrndt, M. M. Malamud and H. Neidhardt, Scattering matrices and Weyl functions, Proc. London Math. Soc. (3) 97 (2008), 568-598.

[8] S. Clark, F. Gesztesy, And M. Mitrea, Boundary Data Maps for Schrödinger Operators on a Compact Interval, Math. Modelling Nat. Phenomena 5, 4 (2010), 73-121.

[9] V. Derkach, S. Hassi, M. Malamud, And H. De Snoo, Boundary relations and their Weyl families, Trans. Amer. Math. Soc. 358 (2006), 5351-5400. 
[10] V. A. Derkach And M. M. Malamud, Generalized resolvents and the boundary value problems for Hermitian operators with gaps, J. Funct. Anal. 95 (1991), 1-95.

[11] V. A. Derkach And M. M. Malamud, The extension theory of Hermitian operators and the moment problem, J. Math. Sci. 73 (1995), 141-242.

[12] N. Dunford And J. T. Schwartz, Linear Operators, Part II: Spectral Theory. Self-Adjoint Operators in Hilbert Space, Wiley, Interscience, New York, 1988.

[13] K.-H. FöRster And M. M. NAFAlska, Nonnegative extensions via embeddings, in Recent Advances in Operator Theory in Hilbert and Krein spaces, J. Behrndt, K.-H. Förster, and C. Trunk (eds.), Operator Theory: Advances and Applications, Vol. 198, Birkhäuser, Basel, 2009, pp. 171-183.

[14] M. Funushima, Dirichlet Forms and Markov Processes, North-Holland, Amsterdam, Kodansha, Tokyo, 1980.

[15] M. Fukushima, Y. Oshima, And M. Takeda, Dirichlet Forms and Symmetric Markov Processes, de Gruyter, Berlin, 1994.

[16] F. Gesztesy, K. A. Makarov, And E. Tsekanovs Kit, An Addendum to Krein's formula, J. Math. Anal. Appl. 222 (1998), 594-606.

[17] F. GeszTESy And M. Zinchenko, Symmetrized perturbation determinants and applications to boundary data maps and Krein-type resolvent formulas, Proc. London Math. Soc. (3) 104 (2012), 577-612.

[18] I. C. Gohberg And M. G. Krein, Introduction to the Theory of Linear Nonselfadjoint Operators, Translations of Mathematical Monographs, Vol. 18, Amer. Math. Soc., Providence, RI, 1969.

[19] V. I. Gorbachuk And M. L. Gorbachuk, Boundary Value Problems for Operator Differential Equations, Mathematics and its Applications, Vol. 48, Kluwer, Dordrecht, 1991.

[20] V. I. Gorbachuk, M. L. Gorbachuk, And A. N. Kochubei, Extension theory for symmetric operators and boundary value problems for differential equations, Ukrain. Math. J. 41 (1989), 11171129.

[21] M. HARMER, Hermitian symplectic geometry and extension theory, J. Phys. A 33 (2000), 9193-9203.

[22] M. HARMER, Hermitian symplectic geometry and the factorization of the scattering matrix on graphs, J. Phys. A 33 (2000), 9015-9032.

[23] M. HARMER, Inverse scattering for the matrix Schrödinger operator and Schrödinger operator on graphs with general self-adjoint boundary conditions, ANZIAM J. 44 (2002), 161-168.

[24] M. HARMER, Inverse scattering on matrices with boundary conditions, J. Phys. A 38 (2005), 48754885 .

[25] A. N. KochuBeI, On extensions of symmetric operators and symmetric binary relations, Math. Notes 17, 1 (1975), 25-28.

[26] T. Kato, Perturbation Theory for Linear Operators, corr. printing of the 2nd ed., Springer, Berlin, 1980.

[27] V. Kostrykin And R. Schrader, Kirchoff's rule for quantum wires, J. Phys. A 32 (1999), 595630 .

[28] V. KostryKin AND R. SCHRADER, Kirchoff's rule for quantum wires. II. The inverse problem with possible applications to quantum computers, Fortschr. Phys. 48 (2000), 703-716.

[29] M. G. KREIN, On Hermitian operators whose deficiency indices are 1, Comptes Rendue (Doklady) Acad. Sci. URSS (N.S.) 43 (1944), 323-326.

[30] M. G. KREIN, On Hermitian operators whose deficiency indices are equal to one. II, Comptes Rendue (Doklady) Acad. Sci. URSS (N.S.) 44 (1944), 131-134.

[31] M. G. KREIN, On a remarkable class of Hermitian operators, Comptes Rendue (Doklady) Acad. Sci. URSS (N.S.) 44 (1944), 175-179.

[32] M. G. KREIN, Concerning the resolvents of an Hermitian operator with the deficiency-index $(m, m)$, Comptes Rendue (Doklady) Acad. Sci. URSS (N.S.) 52 (1946), 651-654.

[33] M. G. KREIN, The theory of self-adjoint extensions of semi-bounded Hermitian transformations and its applications. I, Mat. Sbornik 20 (1947), 431-495 (Russian).

[34] M. G. KREIN, The theory of self-adjoint extensions of semi-bounded Hermitian transformations and its applications. II, Mat. Sbornik 21 (1947), 365-404 (Russian).

[35] M. G. Krein And Ju. L. Smul'JAn, On linear-fractional transformations with operator coefficients, Amer. Math. Soc. Transl. (2) 103 (1974), 125-152. 
[36] E. KREYSZIG, Introductory Functional Analysis with Applications, Wiley and Sons, New York, 1978.

[37] P. Kurasov, Quantum Graphs: Spectral Theory and Inverse Problems, monograph in preparation.

[38] P. KURASOV AND M. NOWACZYK, Geometric properties of quantum graphs and vertex scattering matrices, Opuscula Math. 30, 3 (2010), 295-309.

[39] V. A. MARChEnKo, Some questions in the theory of one-dimensional linear differential operators of the second order, I, Trudy Moskov. Mat. Obšč. 1 (1952), 327-420 (Russian). English transl. in Amer. Math. Soc. Transl., Ser. 2101 (1973), 1-104.

[40] M. A. NAIMARK, On spectral functions of a symmetric operator, Bull. Acad. Sci. URSS. Sér. Math. 7 (1943), 285-296.

[41] M. A. Naimark, Linear Differential Operators. Part II: Linear Differential Operators in Hilbert Space, Ungar, New York, 1968.

[42] M. NowACZY K, Inverse Problems for Graph Laplacians, Ph.D. Thesis, Lund University, 2007.

[43] G. Pólya And G. Szegő, Problems and Theorems in Analysis II, Springer, Berlin, 1976.

[44] M. Reed And B. Simon, Methods of Modern Mathematical Physics. II: Fourier Analysis, SelfAdjointness, Academic Press, New York, 1975.

[45] F. Rofe-Beketov and A. Kohlkin, Spectral Analysis of Differential Operators: Interplay Between Spectral and Oscillatory Properties, World Scientific Monograph Series in Mathematics, Vol. 7, World Scientific Publishing Co. Ptc. Ltd., Singapore, 2005.

[46] B. Simon, Trace Ideals and Their Applications, Mathematical Surveys and Monographs, Vol. 120, 2nd ed., Amer. Math. Soc., Providence, RI, 2005.

[47] J. von Neumann, Allgemeine Eigenwerttheorie Hermitescher Funktionaloperatoren, Math. Ann. 102, 1 (1930), 49-131 (German).

[48] J. Weidmann, Linear Operators in Hilbert Spaces, Graduate Texts in Mathematics, Vol. 68, Springer, New York, 1980.

[49] J. Weidmann, Lineare Operatoren in Hilberträumen. Teil I, Mathematische Leitfäden. Teubner, Stuttgart, 2000.

[50] J. Weidmann, Lineare Operatoren in Hilberträumen. Teil II, Mathematische Leitfäden. Teubner, Stuttgart, 2003.

[51] D. R. YAfaEv, Mathematical Scattering Theory. General Theory, Transl. Math. Monographs, Vol. 105, Amer. Math. Soc., Providence, RI, 1992.

[52] D. R. YAFAEV, Perturbation determinants, the spectral shift function, trace identities, and all that, Funct. Anal. Appl. 41 (2007), 217-236.

[53] A. Zettl, Sturm-Liouville Theory, Mathematical Surveys and Monographs, Vol. 121, Amer. Math. Soc., Providence, RI, 2005. 